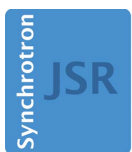

JOURNAL OF SYNCHROTRON RADIATION

ISSN 1600-5775

Received 25 September 2020

Accepted 20 November 2020

Edited by K. Kvashnina, ESRF - The European Synchrotron, France

\# Current address: AGH University of Science and Technology, Krakow, Poland.

$\S$ Current address: School of Physical Science and Technology, Shanghai Tech University,

Shanghai 201210, Popele's Republic of China.

- Current address: University Grenoble Alpes, CNRS, IRD, Irstea, Météo France, OSUG,

FAME, 38000 Grenoble, France.

Keywords: X-ray emission spectrometers; crystal analyzers; Rowland circle; Johann geometry.

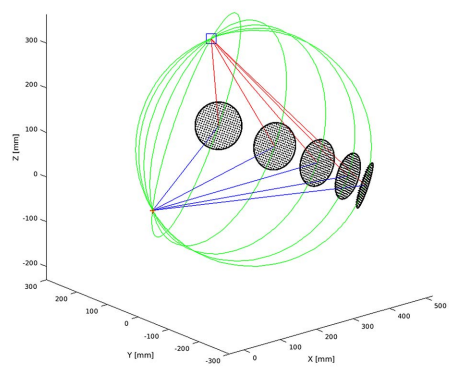

(C) 2021 International Union of Crystallography

\section{The five-analyzer point-to-point scanning crystal spectrometer at ESRF ID26}

\author{
Pieter Glatzel, ${ }^{\mathrm{a} *}$ Alistair Harris, ${ }^{\mathrm{b}}$ Philippe Marion, ${ }^{\mathrm{a}}$ Marcin Sikora, ${ }^{\mathrm{a}} \neq$

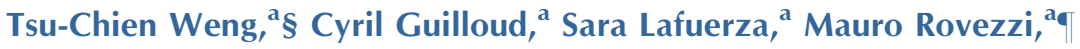 \\ Blanka Detlefs ${ }^{\mathrm{a}}$ and Ludovic Ducotté ${ }^{\mathrm{a}}$
}

aESRF - The European Synchrotron, 71 Avenue des Martyres, 38000 Grenoble, France, and ${ }^{\mathbf{b}}$ Design et Mécanique,
Les Coings, 38210 Montaud, France. *Correspondence e-mail: glatzel@esrf.fr

X-ray emission spectroscopy in a point-to-point focusing geometry using instruments that employ more than one analyzer crystal poses challenges with respect to mechanical design and performance. This work discusses various options for positioning the components and provides the formulas for calculating their relative placement. Ray-tracing calculations were used to determine the geometrical contributions to the energy broadening including the source volume as given by the beam footprint on the sample. The alignment of the instrument is described and examples are given for the performance.

\section{Introduction}

Inner-shell photon-in/photon-out spectroscopy provides an element-selective probe of local coordination and electronic structure (Kotani \& Shin, 2001; de Groot \& Kotani, 2008; Kowalska et al., 2016; Sa, 2014; Bokhoven \& Lamberti, 2016). $\mathrm{X}$-ray emission spectroscopy (XES) can be defined as the detection of X-rays emitted from a sample following core-hole creation in an analyte atom with an instrumental energy bandwidth that is on the order of the core-hole lifetime broadening. We refer to such an energy resolution as 'high' to distinguish it from standard fluorescence measurements using conventional solid-state detectors with a resolving power $E / \Delta E<50$. XES can be performed by taking advantage of various modes of core-hole creation of which the most common is photo-excitation but also ion/electron bombardment and radioactive isotopes (e.g. $K$ capture decay) have been used (Kavčič, 2012; Glatzel et al., 2001). XES following photo-excitation is a second-order optical process that is theoretically treated using the Kramers-Heisenberg equation (Kotani \& Shin, 2001). An instrument for XES thus enables high energy resolution X-ray fluorescence spectroscopy (e.g. $K \beta$ spectroscopy), resonant $\mathrm{X}$-ray emission spectroscopy (RXES), resonant inelastic X-ray scattering (RIXS) and high energy resolution fluorescence detected (HERFD) X-ray absorption spectroscopy (Glatzel et al., 2013; Lafuerza, Retegan et al., 2020). X-ray Raman spectroscopy (Rueff \& Shukla, 2010) does not require continuously tuneable (i.e. scanning) photon-out spectroscopy and is thus ideally carried out with the spectrometer angle fixed close to backscattering in order to optimize the energy resolution and focusing properties (Sokaras et al., 2012; Huotari et al., 2017, 2011; Fister et al., 2006; Schülke, 2007; Mao et al., 2006; Hiraoka et al., 2013).

In the hard X-ray regime instruments based on perfect crystal Bragg optics are generally used to analyze the emitted 
$\mathrm{X}$-rays and thus achieve the required energy resolution. Common geometries are Rowland or von Hámos. The energy of the emitted X-rays is around a fluorescence line or an absorption edge. Following Bragg's law $(\lambda=2 d \sin \theta)$ the reflection angle $\theta$ cannot be chosen freely and is dependent on the atomic number and the electronic transition of interest. An alternative to Bragg optics would be energy-dispersive transition-edge sensors with dramatically reduced energy bandwidth compared with conventional solid-state detectors (Li et al., 2018; Doriese et al., 2017).

Hard XES has a long history dating back to Henry Moseley who used the technique to order elements in the periodic table. The technique has experienced a sharp increase in demand in recent years. This is partly due to the larger number of available hard X-ray beamlines at synchrotron radiation sources around the world that are suitable for XES. Even more important is a better understanding of the theory of XES. User-friendly codes to calculate inner-shell spectra now provide the possibility to model XES data [e.g. FEFF, ORCA, FDMNES, Crispy (Rehr et al., 2010; Neese, 2018; Bunău \& Joly, 2009; Retegan, 2020)]. The full potential of this technique thus becomes available to a broad user community with a wealth of applications.

Non-resonant XES does not require a monochromatic incident beam, which considerably relaxes the constraints on the X-ray source. At a synchrotron radiation source or freeelectron laser one can use a 'pink' beam with an energy bandwidth much larger than the core-hole lifetime broadening (Dikaya et al., 2020). High-quality Bragg optics have become available at a reasonable cost which has led to the development of instruments by many research groups. XES and X-ray absorption spectroscopy (XAS) in the laboratory require very similar instrumentation and recent years have seen a fantastic increase in the application of laboratory X-ray spectroscopy (see the review by Zimmermann et al., 2020). XES finds attractive applications for time-resolved studies at synchrotrons, free-electron lasers and using pulsed laboratory sources (Alonso-Mori et al., 2012, 2015; Szlachetko et al., 2014, 2017; Abraham et al., 2019; Anwar et al., 2019; Wernet, 2019; Tu et al., 2019; Miaja-Avila et al., 2016; Smolentsev et al., 2020; Vankó et al., 2013).

The key components are an X-ray emitting sample that defines the source volume, one or several analyser crystals and a photon detector. Many instruments use bent crystals that are either cylindrical or spherical in shape. The bending radius, together with the Bragg angle $\theta$, defines the relative position between source volume, analyser and detector. In general, a smaller bending radius results in a larger solid angle captured. At the same time, bending of the crystal wafer introduces elastic stress and thus a broader reflectivity curve. Mechanical stress is likely to deform the surface (Honkanen et al., 2014a).

The relation between the energy bandwidth and the spread in Bragg angle is given by the differential form of Bragg's law $\Delta E=E \Delta \theta \cot \theta$, where $\Delta \theta$ is given by the properties of the components and their geometrical arrangement. There are several possibilities to design an instrument for XES and some guidelines must be established. First, a spectrometer may be dispersive or scanning (point-to-point). A dispersive instrument covers a range of Bragg angles in a stationary configuration and the energies are spatially separated on a positionresolving detector. Such an instrument is often mechanically simpler and ideally suited for time-resolved studies as all energies of a spectrum are recorded simultaneously, i.e. a spectrum can be measured in a single shot of X-rays. In this case the energy resolution is determined by the properties of the analyzer crystal and the position resolution of the detector (Kuzmenko et al., 2019; Alonso-Mori et al., 2012; Machek et al., 2007; Dickinson et al., 2008; Kavčič et al., 2012). The influence of the source volume on the energy resolution strongly depends on the chosen geometry. A scanning instrument attempts to use the entire available solid angle, i.e. the crystal analyzer surface, in an energy bandwidth that is sufficiently small to result in spectra that allow for chemical analysis. Here, $\Delta \theta$ is given by the source volume, the properties of the crystal and geometric contributions that strongly depend on the precise positioning of the analyzer crystals relative to the source volume.

Scanning instruments are mechanically challenging particularly when more than one analyzer crystal is employed as the relative displacement between the crystals must be very precise (Sokaras et al., 2013; Uruga et al., 2019; Ablett et al., 2019; Duan et al., 2017; Moretti Sala et al., 2018; Llorens et al., 2012). They do not allow measurement of an entire spectrum in a single exposure but a spectrum is recorded by moving the crystal(s) and detector along a given trajectory. The use of the available solid angle in one energy point and the small detector surface required compared with dispersive setups makes scanning instruments more suitable for measurements of very weak fluorescence signals. Also, HERFD XAS is most efficiently carried out with a scanning instrument as only the intensity in the maximum of the fluorescence line is of interest which should be recorded with the entire available solid angle. We discuss here a scanning multi-crystal X-ray emission spectrometer on beamline ID26 of the European Synchrotron Radiation Facility that was commissioned in 2008. The mechanical design was presented by Ducotté et al. (2010). The concept of the instrument has since then been transferred to other beamlines at the ESRF (BM23, ROBL) and elsewhere (Kleymenov et al., 2011). This paper reports the experiences and lessons learned from working with this instrument. The ray-tracing code was written in MATLAB and is available upon request.

\section{Description of geometry}

The source position $(0,0,0)$ of the spectrometer is defined by the position where the incoming X-ray beam impinges on the sample. In most cases, it is necessary to keep this position fixed, for example when an ion, electron or X-ray beam is used to create the inner-shell vacancy in the sample and the beam cannot be easily moved. If a radioactive isotope is used as an $\mathrm{X}$-ray emitter, this constraint can be relaxed. The source volume of the spectrometer is given by the incident beam size, the orientation of the sample surface with respect to the 
incoming beam direction and the attenuation length of the emitted X-rays. The crystal analyzer has a spherical shape with radius $R_{\mathrm{P}}$. The position of the centre of crystal A, the detector $\mathrm{D}$ and the Rowland circle $\mathrm{K}$ are given by

$$
\mathrm{A}=\left(\begin{array}{c}
A_{x} \\
A_{y} \\
A_{z}
\end{array}\right) ; \mathrm{D}=\left(\begin{array}{c}
D_{x} \\
D_{y} \\
D_{z}
\end{array}\right) ; \mathrm{K}=\left(\begin{array}{c}
K_{x} \\
K_{y} \\
K_{z}
\end{array}\right) .
$$

An index $n(n=-2,-1,0,1,2)$ is introduced to distinguish between the five analyzer crystals. Each crystal $\mathrm{A}_{n}$ describes a Rowland circle $\mathrm{K}_{n}$ with its detector $\mathrm{D}_{n}$. The Rowland circle is defined by its radius $R_{\mathrm{R}}$ that is half the bending radius $R_{\mathrm{P}}=$ $2 R_{\mathrm{R}}$ of the reflecting crystal planes. In Johann geometry, the crystal optical surface has the same radius as the planes of the chosen crystal reflection. The bending radius is assumed to be identical for all five crystals. We note that the manufacturing process may give the best energy resolution for slightly different values for the bending radii of the analyzers $( \pm 5 \mathrm{~mm})$. However, it is undesirable to have all components move on Rowland circles with different diameters because the foci would not coincide on the detector (see discussion of detector below). The instrument thus approximates all circle diameters to identical values. Recent improvements in the crystal analyser manufacturing process result in more and more homogeneous analyser properties.

We define the position $\mathrm{K}_{n}$ of the Rowland circle by the coordinates where a beam from the source position hits the centre of the crystal in a symmetric configuration, i.e. the crystal asymmetry angle $\alpha=0$. We furthermore define a starting configuration for $n=0$ where the crystal is oriented such that the normal on the crystal planes in $\mathrm{K}_{n}$ is parallel to the $x$ axis. The configuration is shown in Fig. 1 for a Bragg angle $\theta_{\mathrm{B}}=75^{\circ}$.

There are several reasons to modify this starting configuration. In case of a crystal asymmetry angle $\alpha$ between the optical surface and the crystal planes, the crystal must be oriented such that the normal on the crystal planes is rotated by $-\alpha$ in the $(x, z)$ plane relative to the starting position in Fig. 1, i.e. counter-clockwise around the $y$ axis. This is to ensure the same Bragg angle as in the symmetric configuration. The source-to-crystal and detector-to-crystal distances are $R_{\mathrm{p}} \sin (\vartheta+\alpha)$ and $R_{\mathrm{p}} \sin (\vartheta-\alpha$ ), respectively (Suortti et al., 1999). The crystal must be offset from the centre position $\mathrm{K}_{n}$ by

$$
a_{z, \alpha}=\frac{R_{\mathrm{p}}}{2} \sin (2 \alpha)
$$

and

$$
a_{x, \alpha}=\frac{R_{\mathrm{p}}}{2}(1-\cos 2 \alpha),
$$

followed by a counter-clockwise rotation of the crystal (i.e. the optical surface) around the $y$ axis by $2 \alpha$.

The positions of the crystal, the Rowland circle and the detector including a possible asymmetry are thus

$$
\begin{aligned}
& \mathrm{A}_{0}=\left(\begin{array}{c}
R_{\mathrm{P}} \sin (\vartheta+\alpha) \sin (\vartheta-\alpha) \\
0 \\
R_{\mathrm{P}} \sin (\vartheta+\alpha) \cos (\vartheta-\alpha)
\end{array}\right), \\
& \mathrm{K}_{0}=\left(\begin{array}{c}
R_{\mathrm{P}} \sin ^{2} \vartheta \\
0 \\
R_{\mathrm{P}} \sin \vartheta \cos \vartheta
\end{array}\right), \\
& \mathrm{D}_{0}=\left(\begin{array}{c}
0 \\
0 \\
2 R_{\mathrm{P}} \sin \vartheta \cos \vartheta
\end{array}\right) .
\end{aligned}
$$

The energy bandwidth is given by intrinsic contributions of the bent crystal and geometric contributions are given by the geometry between the source and crystal. The intrinsic contributions are calculated by considering the elastic bending and the mechanical stress of a crystal wafer with a certain
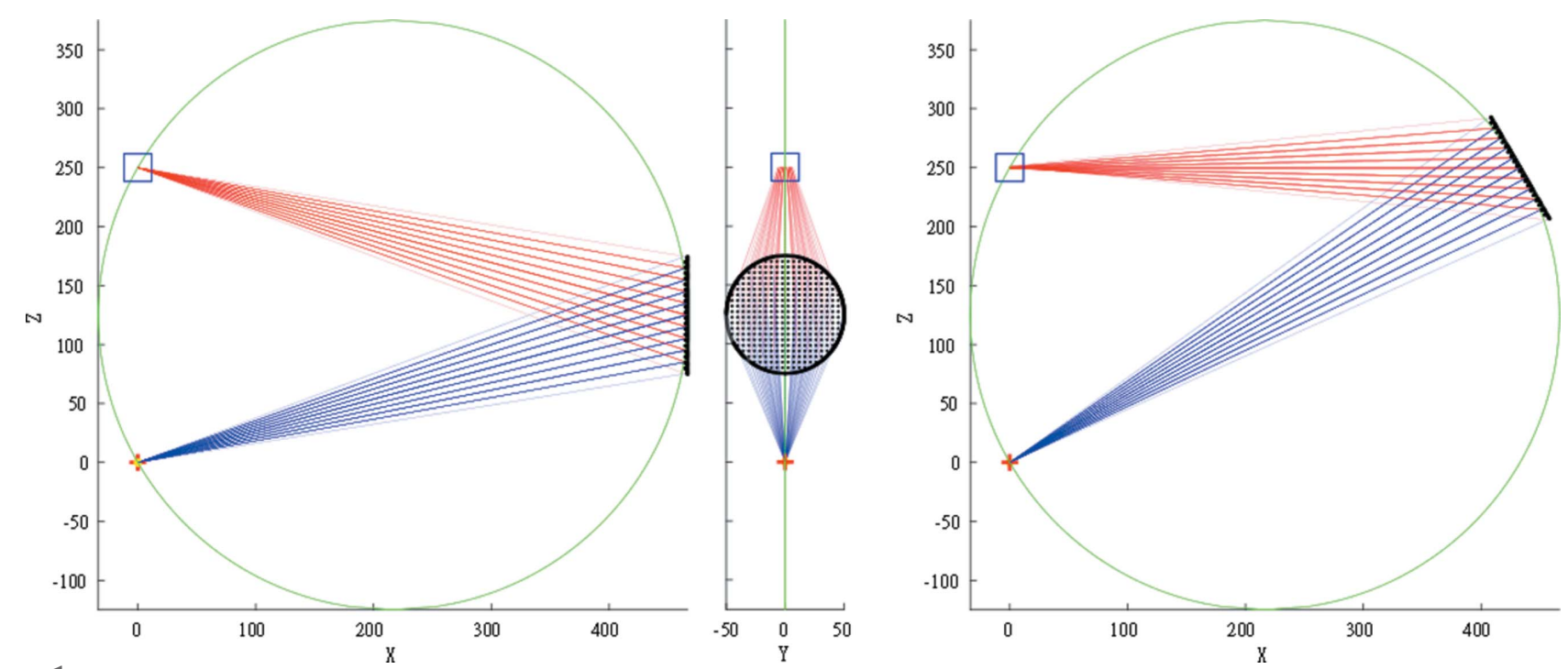

Figure 1

Source, analyzer crystal $(r=50 \mathrm{~mm})$ and detector on a Rowland circle $\left(R_{\mathrm{R}}=250 \mathrm{~mm}, \theta_{\mathrm{B}}=75^{\circ}\right)$. In Johann geometry the reflecting crystal planes and the optical surface have a bending radius $R_{\mathrm{P}}=2 R_{\mathrm{R}}$. The configuration on the right shows the placement of a crystal with a $15^{\circ}$ asymmetry angle. 

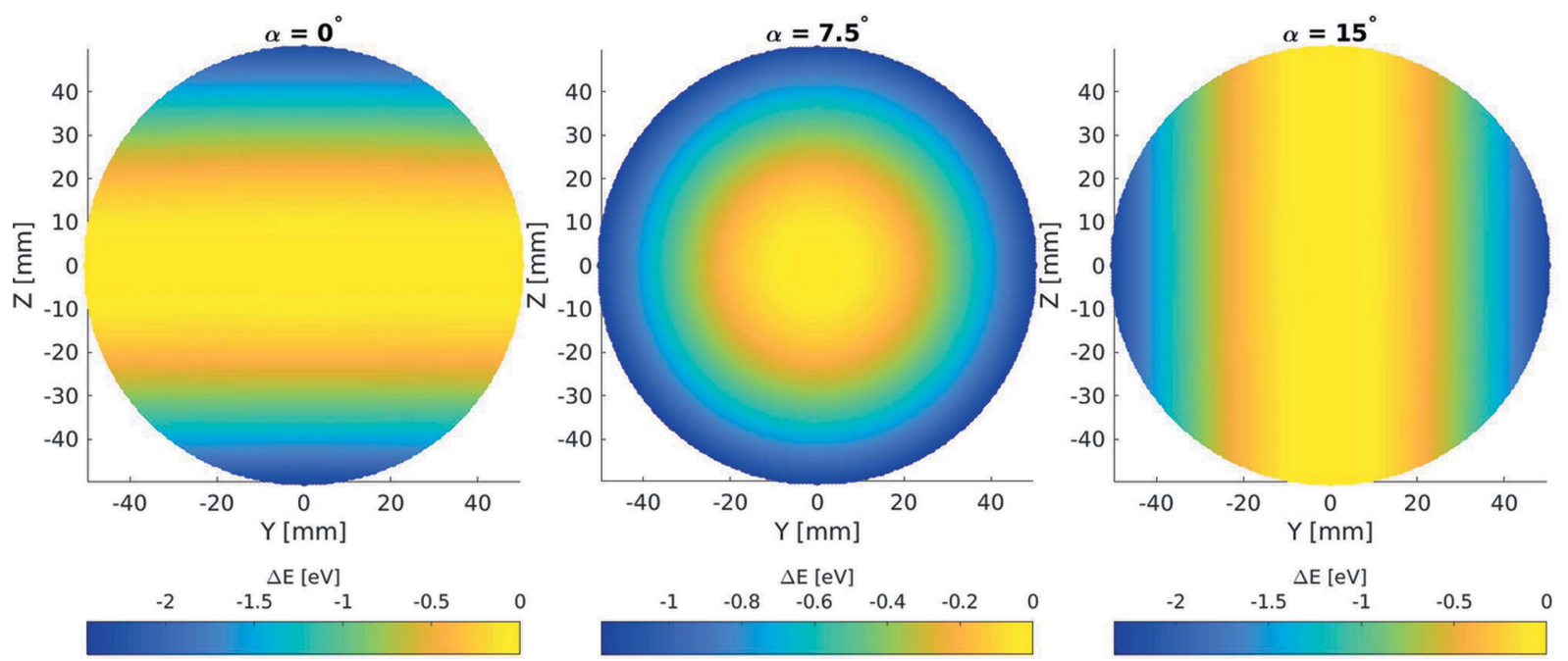

Figure 2

Ray-tracing simulations for $R_{\mathrm{P}}=500 \mathrm{~mm}, r=50 \mathrm{~mm}, \theta_{\mathrm{B}}=75^{\circ}$ (central beam) for the $\mathrm{Si}(440)$ reflection. The images show the crystal surface. The colours indicate the reflected energies. The asymmetry angles are $0^{\circ}, 7.5^{\circ}$ and $15^{\circ}$ from left to right. The deviations $(\Delta E)$ are given in the colourbar in electronvolts.

thickness and radius (Honkanen et al., 2014a). Formulas for geometric contributions have been given by several authors (Bergmann \& Cramer, 1998; Moretti Sala et al., 2018). In general, all geometric contributions increase with decreasing Bragg angle and therefore crystal material and Bragg angle are chosen such that the Bragg angle is close to $90^{\circ}$. Here we determine the bandwidth by means of ray-tracing simulations and do not attempt to obtain the energy bandwidth using analytical formulas. We only note that the Johann error in energy scales with $\cot ^{2}\left(\vartheta_{\mathrm{B}}+\alpha\right)$ (Suortti et al., 1999) and it is thus interesting to study how the Johann error for a given Bragg angle changes with the crystal asymmetry. Fig. 2 shows the deviation $(\Delta \theta)$ from the Bragg angle of the central beam $\theta_{\mathrm{B}}$ across the surface of the analyzer crystal. In the absence of asymmetry the deviation shows the typical dependence for the Johann error in the vertical scattering plane. An asymmetry of $15^{\circ}$ eliminates the Johann error as $\theta_{\mathrm{B}}+\alpha=90^{\circ}$ (Moretti Sala et al., 2018), but the contribution from the crystal surface outside the scattering plane (i.e. the sagittal direction) increases by the same amount as the decrease in Johann error (Fig. 2). If the asymmetry is chosen as $\left(90-\theta_{\mathrm{B}}\right) / 2$ we obtain a radial distribution of $\Delta \theta$ on the surface. The energy bandwidth calculated by including $80 \%$ of all rays around $\theta_{\mathrm{B}}$ is $1.14 \mathrm{eV}$ for the $\mathrm{Si}(440)\left(R_{\mathrm{P}}=500 \mathrm{~mm}\right)$ reflection at $75^{\circ}(6684.9 \mathrm{eV})$ for $\alpha=0^{\circ}$ and $15^{\circ}$ and $0.94 \mathrm{eV}$ for $\alpha=7.5^{\circ}$. The figure nicely shows how placing a mask in front of the crystal improves the energy resolution. We note that the figure errors of the crystal surface may make a significant contribution to the geometrical energy broadening and they may increase with increasing radius $r$. In this case, a mask will help to select the part of the crystal surface with the smallest figure error. A toroidal analyser shape could reduce or eliminate the energy broadening outside the scattering plane (Bryzgunov, 2000; Jahrman et al., 2019). However, the required bending radius in the sagittal plan depends on the Bragg angle (see below).
A finite source size will contribute to the geometrical energy bandwidth depending on the Bragg angle (Bergmann \& Cramer, 1998). In standard configuration on ID26 the incoming beam with size $0.1 \mathrm{~mm}$ (vertical) $\times 0.7 \mathrm{~mm}$ (horizontal) (values before the upgrade of ESRF to the extremely brilliant source) impinges on the sample under $45^{\circ}$ and the horizontal scattering angle with the central crystal is $90^{\circ}$. In this case, the geometrical contribution increases from $0.3 \mathrm{eV}$ to $0.5 \mathrm{eV}$ for the $\mathrm{Si}(440)$ reflection at $6684.9 \mathrm{eV}\left(R_{\mathrm{P}}=\right.$ $1000 \mathrm{~mm}$ ). When reducing the bending radius to $R_{\mathrm{P}}=500 \mathrm{~mm}$ the bandwidth increases to $1.1 \mathrm{eV}$ and $1.6 \mathrm{eV}$ for the two cases. The chosen beam size and bending radius are often a compromise between spectrometer energy resolution and possible radiation damage in the sample. We note that the source size contribution can be minimized in some dispersive setups with 2D detectors (Holden et al., 2017; Nowak et al., 2020; Kavčič et al., 2012)

In order to cover a large solid angle it is necessary to use more than one analyzer crystal. One could position several crystals on one Rowland circle each with an appropriate asymmetry angle corresponding to its offset $a_{z, \alpha}$. We note that placing a crystal off the Rowland centre $\mathrm{K}$ without an asymmetric cut increases the energy bandwidth. At $\theta_{\mathrm{B}}=75^{\circ}$ moving the crystal up (down) from the symmetric position by $50 \mathrm{~mm}$ with the crystal surface centre on the Rowland circle increases the geometrical energy bandwidth from $0.3 \mathrm{eV}$ to $1.2 \mathrm{eV}$ $(1.6 \mathrm{eV})$ for the $\mathrm{Si}(440)$ reflection at $6684.9 \mathrm{eV}\left(R_{\mathrm{P}}=\right.$ $1000 \mathrm{~mm})$.

The least complex way to increase the solid angle is to add crystals in the sagittal plane (Fig. 3). We use a rotation around the $z$ axis $\mathcal{R}_{z}$ to achieve an offset between the crystals along the $y$ axis. Each crystal defines a Rowland circle. The five circles intersect at two points. One intersection point coincides with the source volume. The detector may be placed at the second intersection point. We would like the sagittal offset 

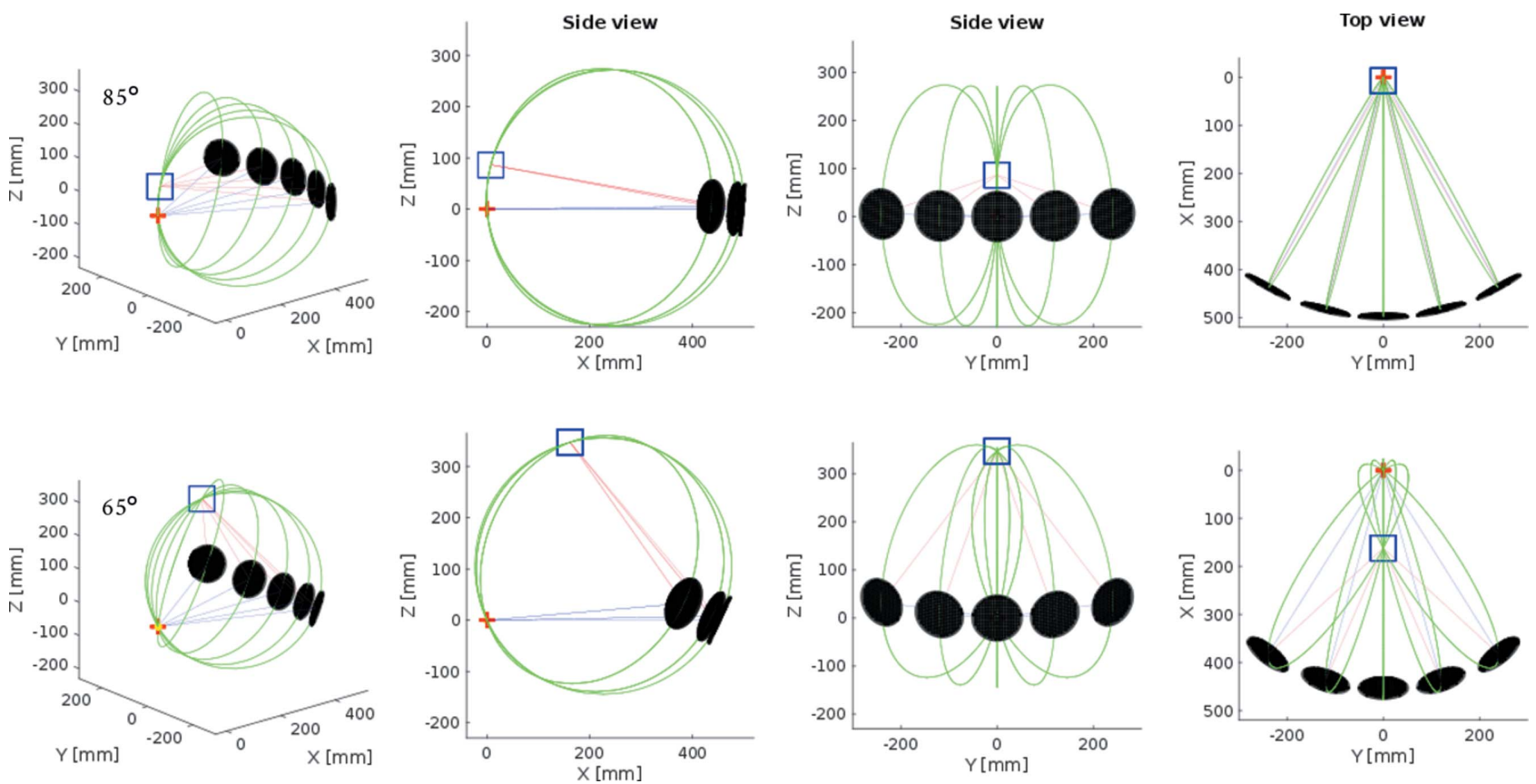

Figure 3

Analyzer crystal and detector positions for $\theta=85^{\circ}$ and $\theta=65^{\circ}\left(R_{\mathrm{P}}=500 \mathrm{~mm}, a_{z, \text { off }}=0, a_{y, \text { off }}=120 \mathrm{~mm}, \alpha=0^{\circ}\right)$. Each analyzer defines its own Rowland circle. Consequently, there are five meridional planes. Note that since only the central crystal remains at a fixed vertical position, only the centre cradle is rotated to the Bragg angle $\theta_{\mathrm{B}}$. For the outer crystals, the Bragg angle is a combination of cradle rotation and vertical displacement.

between the crystals to be as small as possible and constant for all Bragg angles. This is desirable because in situ and highpressure cells often have limited opening windows. The ratio between the solid angle covered by the analyzer crystals and the solid angle given by the required window opening such that the source volume sees all crystals should be as large as possible. The rotation angle is given by

$$
\gamma_{z}=\arcsin \left(\frac{a_{y, \text { off }}}{A_{0, x}}\right) \text {. }
$$

The spectrometer on ID26 uses $a_{y, \text { off }}=120 \mathrm{~mm}$ with a $100 \mathrm{~mm}$ diameter for the analyzer crystals.

Finally, we would like to have the central analyzer position at $a_{z, \text { off }}=0$ for all Bragg angles. This is again desirable for sample environments with limited opening windows but also to ensure that the scattering geometry varies as little as possible over a scan range of the Bragg angle. This is also important for isotropic X-ray fluorescence as a strong background may arise from Compton scattering that is minimized at $90^{\circ}$ scattering angle for linear polarized incoming light. We thus rotate all analyzer crystals around the $y$ axis $\left(\mathcal{R}_{y}\right)$ by an angle of

$$
\gamma_{y}=\frac{\pi}{2}-\vartheta+\alpha-\arcsin \left(\frac{a_{z, \text { off }}}{R_{\mathrm{P}} \sin (\vartheta+\alpha)}\right)
$$

where we give the general expression for any position $a_{z, \text { off }}$ of the centre of the crystal analyzer along the $z$ axis.

The detector positioned on the Rowland circles provides the smallest focal spot size in the direction of the scattering plane but a large spot size in the sagittal direction (the condition for meridional focusing is $R \sin \vartheta$ for the image-tomirror distance for a spherical mirror). For $\theta_{\mathrm{B}}=75^{\circ}$ the focus is $13 \mathrm{~mm}$-wide in the $y$ direction for $\alpha=0^{\circ}$ and $20 \mathrm{~mm}$ for $\alpha=$ $15^{\circ}$. The sagittal spot size decreases with increasing Bragg angle but does not depend on the crystal bending radius $R_{\mathrm{P}}$.

The condition for sagittal focusing is $\left(R_{\mathrm{P}} \sin \vartheta\right) /(-\cos 2 \vartheta)$ for the distance between the crystal and detector. We can use this in case we would like the analyzer crystals to give distinct foci on the detector. Images of the foci for a five-crystal spectrometer are shown in Fig. 4. Moving the detector out of the Rowland circle along the direction defined by $A_{0}$ and the Rowland circle intersection point separates the foci from the five crystals and changes the focal shape. This requires a larger detector area or five separate detectors (Moretti Sala et al., 2018). The images also show that the position resolution of the detector can be used to correct the energy dispersion and thus improve the resolution. For example, this was done by Huotari et al. (2005) and Honkanen et al. (2014b) for a Johann spectrometer.

The spectrometer on ESRF ID26 currently uses a singleelement detector (avalanche photo diode, silicon drift diode) such that continuous scans with acquisition times down to $10 \mathrm{~ms}$ per datapoint are easily achievable and the data acquisition chain is simple. The rapid development of pixel detectors make them a very attractive alternative also for continuous scans in spectroscopy and ID26 may implement one in the future. We note that it is beneficiary to have only a small detector surface (or a carefully chosen region of interest on the 2D detector) exposed if the background must be kept low. Stray X-rays in the experimental hutch may give rise to a signal as strong as or stronger than weak fluorescence lines. 

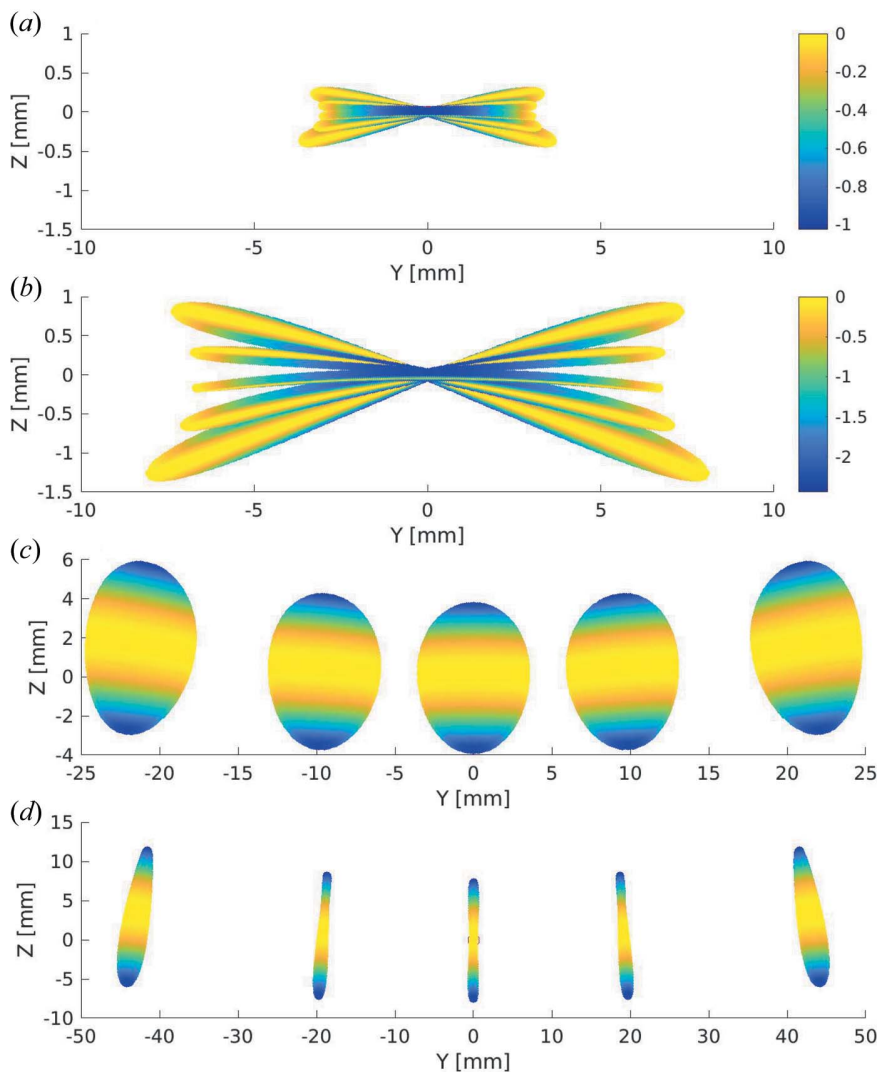

Figure 4

Ray-tracing images on the detector for a point source in a five-crystal spectrometer with $R_{\mathrm{P}}=500 \mathrm{~mm}, r=50 \mathrm{~mm}, \alpha=0^{\circ}$ for $(a) \theta=80^{\circ},(b)-(d)$ $\theta=75^{\circ}$. The detector was placed $(a)-(b)$ on the Rowland circle and offset by (c) $37.35 \mathrm{~mm}$ and $(d) 74.7 \mathrm{~mm}$ outside the Rowland circle which gives the maximal sagittal focusing for the central crystal. The colours show the energy deviation (in electronvolts) from the central energy $6684.9 \mathrm{eV}$. The colour bar in (b) also applies to $(c)$ and $(d)$.

This is particularly important if the analyte atom is low in concentration or in a matrix of high- $Z$ elements that strongly fluoresce. In this case or when a lower-order crystal analyzer reflection is relevant, energy resolution of the spectrometer detector (e.g. using a silicon drift diode detector) may help to further reduce the background. A 2D detector also provides the possibility to record the X-ray background outside the focus region. However, the background underneath the fluorescence line of interest may be different inside the focus than outside the focus. The reason is that some of the background is Bragg reflected (e.g. Compton scattering or tails of strong fluorescence lines emitted from the matrix) and is thus only observed in the focus.

The ID26 spectrometer uses a slit in front of the detector with a $1.0-1.5 \mathrm{~mm}$ opening in the meridional direction. The slit defines the position of the detector on the Rowland circle. Having all five crystals impinge on the same detector element puts additional constraints on the alignment procedure that we explain in the following. Closing the slits may improve the energy resolution at the expense of intensity. However, if the slits cut into the focus the spectrometer becomes sensitive to imperfect tracking during the scan and a slit size above $1 \mathrm{~mm}$ has proven to be a good choice.

\section{Spectrometer alignment and scanning}

Each analyzer crystal has four degrees of freedom. In the bottom is a translation along $X$ that carries a rotation stage with a vertical axis. On top of this rotation state is a vertical translation to achieve $A_{z}$ that carries a cradle to achieve a rotation with the axis perpendicular to the meridional plane of the analyzer (Fig. 3). The five assemblies for each crystal are separated by $a_{y, \text { off }}$. The stages serve the alignment and scanning of the spectrometer. The motion errors must be kept to a minimum in order to avoid misalignment of the meridional angle between the assemblies. For the design of the instrument the objective was to keep the error in the meridional angle below $10 \mu \mathrm{rad}$ over a scan range. All 20 crystal stages are mounted on a granite block that can be displaced along $X$ in order to accommodate bending radii between $0.5 \mathrm{~m}$ and $2 \mathrm{~m}$. The detector is mounted on translation stages and a rotation stage. It is positioned with a precision of $50 \mu \mathrm{m}$ and the detector slit always faces the central crystal.

The spatial coordinates of all components and the sample position are determined with a precision of $\sim 100 \mu \mathrm{m}$ by an optical alignment. The source volume is defined by aligning the incoming beam in $X$ and $Z$ through a pinhole. The alignment along $Y$ is less critical and is achieved by considering the dimensions of the sample mount. A reference is placed in the source volume and the five analyzer crystals are aligned such that the fluorescence signal is centred on the detector slit, i.e. we do not attempt to maximize the fluorescence signal in this step. Thus, the Bragg angle and therefore the spectrometer energy calibration is defined by the geometrical alignment and not by the presumed maximum of the fluorescence line, the energy of which is not always precisely known. This approach gives the best results for tracking of the focal spot over a given scan range.

In a second step, the elastic peak is measured for all crystals and the $A_{z}$ position for each crystal is adjusted such that the energies of all elastic peaks coincide. Alternatively, the meridional angle for each crystal can be adjusted. There are several reasons why a geometrical alignment does not achieve identical energy calibration between crystals. An error in the optical alignment translates into an error of the Bragg angle and thus the energy. The most sensitive direction is $Z$. Furthermore, the crystals may have a small asymmetry angle while we assume a symmetric geometry in the alignment. The two errors are related in the Rowland geometry as we discussed before and the two corrections are thus equivalent. An unintended miscut is $<0.05^{\circ}$ according to the manufacturers and a misalignment in $Z$ will be less than a few hundred micrometres. In both cases, the energy resolution will not suffer. The final step of the alignment is a scan of the detector slit closed to $0.1 \mathrm{~mm}$ along $Z$ to determine the shape of the focus. The appropriate slit size is chosen accordingly. An offset in the vertical direction is introduced for the calculation of the detector position to center the slit in the measured position. This offset is typically a few hundred micrometres and arises from the relative energy calibration between the analyzer crystals. 
The Bragg angle could be calibrated using the energy calibration of the incident beam by measuring the elastic peak. This has led to poor results with respect to tracking over the whole scan range in our experience. Determination of the Bragg angle via the optically aligned spectrometer components leads to better tracking. One reason for this may be an error in the calibration of the energy of the incoming beam. The generally accepted values for absorption edges are often a convention rather than precisely determined quantities. Furthermore, calculation of the Bragg angle from a given wavelength requires precise knowledge of the crystal $d$-spacing. For pure $\mathrm{Si}$ this is known to very high precision but the bonding process of the wafer on the glass substrate may affect the crystal lattice. Furthermore, Ge another important material for analyzer crystals - is not available in such high purity and the manufacturer may not be able to provide the precise

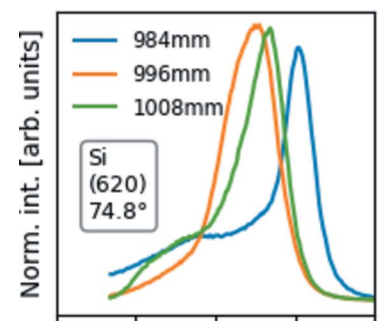

$747574767477 \quad 7478 \quad 7479$ Incoming energy [eV]

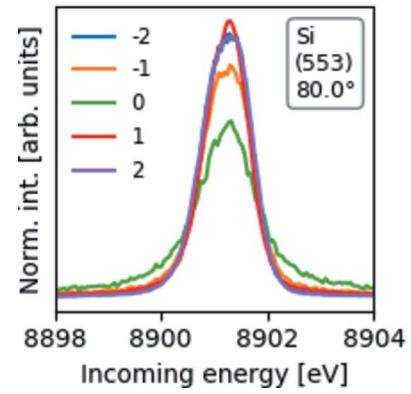

Figure 5

Spectrometer alignment scans for crystals with $r=50 \mathrm{~mm}$ and substrate radius $R_{\mathrm{P}}=1000 \mathrm{~mm}$. Crystal wafer material, reflection, Bragg angle and crystal number (when relevant) are given. The scattering angle is $90^{\circ}$ for crystal $n=0$ in the horizontal plane except for $\mathrm{Si}(620)$ where it is $127^{\circ}$ (top row). Elastic peaks for different Rowland circle radii (top left); elastic peaks for all five crystals at the optimized Rowland circle radius of $996 \mathrm{~mm}$ before (top centre) and after (top right) energy calibration between the crystals. Elastic peaks for different crystal reflections and Bragg angles (bottom row). The increased width of central crystal $n=0$ can be attributed to the angular dependence of the elastic scattering that emphasizes the outer radius of the crystal analyzer surface. value for the lattice parameters.

Although it would be possible to determine the lattice parameters, we note that accurate absolute values for the emission energy are hardly ever needed and ID26 decided to keep the energy calibration of the incident beam and of the XES instrument separate and use the measurement of the elastic peak to link the two.

A stable relative energy calibration is crucial in order to detect small changes in the samples. To this end, the $\theta$-cradles and $A_{z}$ translations are equipped with encoders and the mechanical design ensures very high repeatability. The main source of error is a vertical beam motion on the sample because it modifies the Bragg angle to first order. Slits placed in front of the sample that define the incoming beam position keep this error below $10 \mu \mathrm{m}$. For Fe $K \alpha$ measured using the $\mathrm{Ge}(440)$ reflection at $75^{\circ}$ we determined an error for the energy calibration to less than $10 \mathrm{meV}$ (Lafuerza, Carlantuono et al., 2020). When changing samples the correct placement along $Y$ is achieved by scanning the sample in the $Y$ direction while observing the signal on the detector. Such a scan moves the focus across the detector slit in the sagittal direction and allows alignment to within $100 \mu \mathrm{m}$.

The alignment steps with X-rays are shown in Fig. 5 for $\mathrm{Si}(620)$ crystals. Scans of the elastic peak are shown for further crystal reflections. The top panel shows scans to determine the crystal bending radius $R_{\mathrm{P}}$. We usually take ten scans for different bending radii and use a figure of merit combining energy bandwidth and intensity of the elastic peak to determine the optimal radius. The nominal radius of the glass substrate is $R_{\mathrm{P}}=1000 \mathrm{~m}$ but the best performance is found for
$R_{\mathrm{P}}=996 \mathrm{~mm}$ in this case. The best performance is typically found in a range $\pm 5 \mathrm{~mm}$ around the nominal radius. In general, similar values are found for crystals from the same manufacturing batch and the spectrometer is run using the experimentally determined best radius for all crystals. The elastic peaks differ slightly for each crystal.

The crystals are aligned to give identical centre energies at the half maxima. The energy bandwidths of the elastic peaks for $\mathrm{Si}(620)$ at $74.8^{\circ}$ are between $0.7 \mathrm{eV}$ and $0.8 \mathrm{eV}$ using the $\mathrm{Si}(311)$ reflection for the incident beam. The resolving power of the $\mathrm{Si}(311)$ reflection is $\sim 35700$ and combined with the incident beam vertical divergence we obtain $0.3 \mathrm{eV}$ for the incoming beam energy bandwidth. This gives an energy bandwidth of $0.6(0.7) \mathrm{eV}$ for the spectrometer taking $0.7(0.8) \mathrm{eV}$ for the elastic peak. The calculated geometrical contribution is $0.55 \mathrm{eV}$ in this case and the intrinsic contributions to the crystal analyzer energy broadening are thus small. The elastic peaks for $\operatorname{Si}(620)$ at $74.8^{\circ}$ show a strong asymmetry whereas the elastic peaks for other reflections at higher Bragg angles are more symmetric.

We also tested the dependence of the energy bandwidth on the azimuthal rotation [see also the work by Mortensen \& Seidler (2017)]. This dependence mainly arises from errors in the bonding process such that the wafer is not bent to a perfect sphere. Azimuthal alignment results are shown in Fig. 6 for $\mathrm{Si}(440)$ and $\mathrm{Ge}(620)$ crystals. While Si crystals typically show little influence, Ge crystals may show a strong dependence on the azimuthal angle [in the $\mathrm{Ge}(620)$ crystal example, azimuthal orientation a 2 clearly yields the best performance]. 

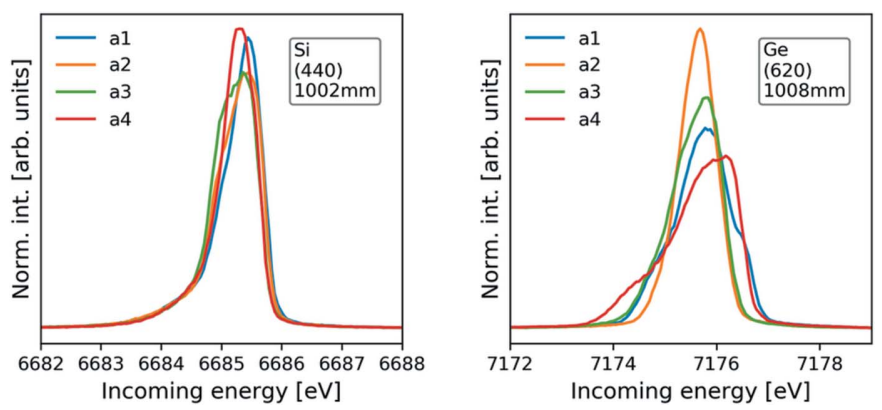

Figure 6

Azimuthal alignment scans for crystals with $r=50 \mathrm{~mm}$ and substrate nominal radius $R_{\mathrm{P}}=1000 \mathrm{~mm}$. Crystal wafer material, reflection and experimentally determined best radius used for the azimuthal alignment are given. The azimuthal alignments were always performed at a Bragg angle of $75^{\circ}$ and, in order to enhance the elastic contribution, at a horizontal scattering angle of $130^{\circ}(\operatorname{crystal} n=0)$. Elastic peaks are shown for four different azimuthal orientations at $45^{\circ}$ intervals (a1, a2, a3, a4). Left: $\mathrm{Si}(440)$ crystal at position $n=0$. Right: $\mathrm{Ge}(620)$ crystal at position $n=-2$.

Table 1 lists the average elastic peak widths as collected from several experimental sessions. The variation around the given values is large because each crystal is different due to the manufacturing process. In particular, crystals made of $\mathrm{Ge}$ show a very large spread with variations up to $40 \%$. The estimated analyzer bandwidth is based on several assumptions (Gaussian shapes, approximate vertical divergence for incoming beam) that have not been verified experimentally. The given values are thus only a very rough guide. The elastic peaks become sharper with increasing Bragg angle and decreasing energy. Otherwise, the bandwidth strongly depends on the crystal quality. The performance of the crystal analyzer has dramatically improved over the past ten years, considerably reducing the energy bandwidth of the spectrometer. This process is ongoing. New fabrication processes for $\mathrm{Ge}$ and the use of other materials (e.g. quartz, $\mathrm{LiNbO}_{3}$ ) will further improve the energy resolution for a given emission energy. Table 1 is therefore only a snapshot of the current situation.

The geometrical energy broadening strongly depends on the crystal bending radius. The spectrometer on ID26 can thus accommodate crystals with radii between $0.5 \mathrm{~m}$ and $2 \mathrm{~m}$. This allows optimization of either the captured solid angle or the energy resolution. The required energy resolution depends on the natural widths of the fluorescence line as discussed in detail by Rovezzi et al.(2017). Crystals with $R_{\mathrm{P}}=500 \mathrm{~mm}$ have pushed the detection limit for the study of $\mathrm{Hg}$ in natural samples below the ppm level (Manceau et al., 2016).

The spectrometer is implemented in the beamline software as a virtual motor and the user only needs to select an emission energy to move all spectrometer motors. RIXS and constant final state scans are executed with single commands after choosing the energy range and step sizes. The XES instrument on ID26 is used in almost all experiments. In order to allow fast change between analyzer crystals, the alignment procedure was automated as much as possible reducing the time for aligning five crystals to below $1 \mathrm{~h}$.
Table 1

Experimental elastic peak energy widths and estimated energy bandwidths (full width at half-maximum) for crystal analyzers with $R_{\mathrm{P}}=$ $1000 \mathrm{~mm}$ and $r=50 \mathrm{~mm}$. A Gaussian profile was assumed for the incoming beam and the spectrometer energy bandwidth. A vertical divergence for the incoming beam of 10 (5) $\mu \mathrm{rad}$ below (above) $9000 \mathrm{eV}$ was considered for the fundamental (third) undulator harmonic.

\begin{tabular}{|c|c|c|c|c|c|}
\hline $\begin{array}{l}\text { Energy } \\
(\mathrm{eV})\end{array}$ & $\begin{array}{l}\text { Monochromator } \\
\text { crystal reflection }\end{array}$ & $\begin{array}{l}\text { Analyzer } \\
\text { crystal } \\
\text { reflection }\end{array}$ & $\begin{array}{l}\text { Analyzer } \\
\text { crystal } \\
\text { Bragg } \\
\text { angle }\left(^{\circ}\right)\end{array}$ & $\begin{array}{l}\text { Elastic } \\
\text { peak } \\
\text { width } \\
(\mathrm{eV})\end{array}$ & $\begin{array}{l}\text { Estimated } \\
\text { analyzer } \\
\text { bandwidth } \\
(\mathrm{eV})\end{array}$ \\
\hline 19965 & $\operatorname{Si}\left(\begin{array}{lll}1 & 1 & 1\end{array}\right)$ & $\operatorname{Si}\left(\begin{array}{lll}12 & 12 & 0\end{array}\right)$ & 76.00 & 5.2 & 4.1 \\
\hline 19608 & $\operatorname{Si}\left(\begin{array}{lll}1 & 1 & 1\end{array}\right)$ & $\operatorname{Si}\left(\begin{array}{lll}12 & 12 & 0\end{array}\right)$ & 81.10 & 5.0 & 3.9 \\
\hline 12496 & $\operatorname{Si}\left(\begin{array}{lll}3 & 1 & 1\end{array}\right)$ & $\operatorname{Ge}(880)$ & 82.80 & 1.3 & 1.2 \\
\hline 10839 & $\operatorname{Si}\left(\begin{array}{lll}3 & 1 & 1\end{array}\right)$ & $\mathrm{Ge}(844)$ & 82.11 & 1.1 & 1.0 \\
\hline 9713 & $\operatorname{Si}\left(\begin{array}{lll}3 & 1 & 1\end{array}\right)$ & 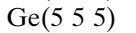 & 77.69 & 1.1 & 1.0 \\
\hline 9441 & $\operatorname{Si}\left(\begin{array}{lll}1 & 1 & 1\end{array}\right)$ & $\mathrm{Ge}\left(\begin{array}{lll}6 & 6 & 0\end{array}\right)$ & 80.00 & 1.7 & 1.1 \\
\hline 9440 & $\mathrm{Si}\left(\begin{array}{lll}3 & 1 & 1\end{array}\right)$ & $\mathrm{Ge}\left(\begin{array}{lll}6 & 6 & 0\end{array}\right)$ & 80.00 & 1.2 & 1.1 \\
\hline 8903 & $\operatorname{Si}\left(\begin{array}{lll}3 & 1 & 1\end{array}\right)$ & $\operatorname{Si}\left(\begin{array}{lll}5 & 5 & 3\end{array}\right)$ & 80.00 & 1.1 & 1.0 \\
\hline 8048 & $\operatorname{Si}\left(\begin{array}{lll}3 & 1 & 1\end{array}\right)$ & $\operatorname{Si}(444)$ & 79.30 & 0.7 & 0.6 \\
\hline 7481 & $\mathrm{Si}\left(\begin{array}{lll}3 & 1 & 1\end{array}\right)$ & $\operatorname{Si}\left(\begin{array}{lll}6 & 2 & 0\end{array}\right)$ & 74.80 & 0.8 & 0.7 \\
\hline 7481 & $\operatorname{Si}\left(\begin{array}{lll}1 & 1 & 1\end{array}\right)$ & $\operatorname{Si}\left(\begin{array}{lll}6 & 2 & 0\end{array}\right)$ & 74.80 & 1.7 & 1.3 \\
\hline 7060 & $\operatorname{Si}\left(\begin{array}{lll}3 & 1 & 1\end{array}\right)$ & $\mathrm{Ge}\left(\begin{array}{lll}6 & 2 & 0\end{array}\right)$ & 79.00 & 0.9 & 0.8 \\
\hline 7060 & $\operatorname{Si}\left(\begin{array}{lll}1 & 1 & 1\end{array}\right)$ & $\operatorname{Ge}\left(\begin{array}{lll}6 & 2 & 0\end{array}\right)$ & 79.00 & 1.2 & 0.6 \\
\hline 6931 & $\mathrm{Si}\left(\begin{array}{lll}3 & 1 & 1\end{array}\right)$ & $\operatorname{Si}\left(\begin{array}{lll}5 & 3 & 1\end{array}\right)$ & 77.00 & 0.6 & 0.5 \\
\hline 6500 & $\operatorname{Si}\left(\begin{array}{lll}1 & 1 & 1\end{array}\right)$ & $\operatorname{Si}(440)$ & 84.10 & 1.0 & 0.4 \\
\hline 6492 & $\operatorname{Si}\left(\begin{array}{lll}3 & 1 & 1\end{array}\right)$ & $\mathrm{Ge}(4 \quad 40)$ & 72.70 & 0.8 & 0.7 \\
\hline 6406 & $\operatorname{Si}\left(\begin{array}{lll}3 & 1 & 1\end{array}\right)$ & $\mathrm{Ge}\left(\begin{array}{lll}4 & 4 & 0\end{array}\right)$ & 75.40 & 0.6 & 0.5 \\
\hline 6272 & $\operatorname{Si}\left(\begin{array}{lll}3 & 1 & 1\end{array}\right)$ & $\mathrm{Ge}\left(\begin{array}{lll}4 & 4 & 0\end{array}\right)$ & 81.20 & 0.6 & 0.5 \\
\hline 5887 & $\mathrm{Si}\left(\begin{array}{lll}3 & 1 & 1\end{array}\right)$ & $\mathrm{Ge}\left(\begin{array}{lll}3 & 3 & 3\end{array}\right)$ & 75.30 & 0.7 & 0.7 \\
\hline 5851 & $\operatorname{Si}\left(\begin{array}{lll}1 & 1 & 1\end{array}\right)$ & $\mathrm{Ge}\left(\begin{array}{lll}3 & 3 & 3\end{array}\right)$ & 76.70 & 1.0 & 0.6 \\
\hline 5846 & $\mathrm{Si}\left(\begin{array}{lll}3 & 1 & 1\end{array}\right)$ & $\mathrm{Ge}\left(\begin{array}{lll}3 & 3 & 3\end{array}\right)$ & 76.90 & 0.6 & 0.5 \\
\hline 5637 & $\mathrm{Si}\left(\begin{array}{lll}3 & 1 & 1\end{array}\right)$ & $\operatorname{Si}\left(\begin{array}{lll}4 & 2 & 2\end{array}\right)$ & 82.80 & 0.4 & 0.3 \\
\hline 5633 & $\mathrm{Si}\left(\begin{array}{lll}3 & 1 & 1\end{array}\right)$ & $\operatorname{Si}\left(\begin{array}{lll}4 & 2 & 2\end{array}\right)$ & 83.10 & 0.5 & 0.4 \\
\hline 5034 & $\operatorname{Si}\left(\begin{array}{lll}1 & 1 & 1\end{array}\right)$ & $\operatorname{Si}\left(\begin{array}{lll}3 & 3 & 1\end{array}\right)$ & 81.30 & 0.7 & 0.3 \\
\hline 4952 & $\operatorname{Si}\left(\begin{array}{lll}3 & 1 & 1\end{array}\right)$ & $\operatorname{Ge}\left(\begin{array}{lll}3 & 3 & 1\end{array}\right)$ & 74.70 & 0.6 & 0.6 \\
\hline 4839 & $\operatorname{Si}\left(\begin{array}{lll}3 & 1 & 1\end{array}\right)$ & $\mathrm{Ge}\left(\begin{array}{lll}3 & 3 & 1\end{array}\right)$ & 80.80 & 0.5 & 0.5 \\
\hline 4511 & $\operatorname{Si}\left(\begin{array}{lll}3 & 1 & 1\end{array}\right)$ & $\mathrm{Ge}\left(\begin{array}{lll}4 & 0 & 0\end{array}\right)$ & 76.30 & 0.8 & 0.8 \\
\hline
\end{tabular}

\section{Outlook}

The complexity of a point-to-point, scanning multi-analyzer XES instrument is manageable. The instrument described in this paper employs spherical Johann crystals that are nowadays available in very high quality at affordable prices from various suppliers. A new concept for a multi-crystal point-topoint spectrometer is presented in a recent paper by Rovezzi et al. (2020). This new design is based on cylindrical crystals and opens up a path towards increasing the captured solid angle while maintaining the energy resolution. It also allows the use of fewer crystal reflections in the hard X-ray range because it can employ Johansson-type crystals. With a growing interest in the scientific community such XES instruments may also be developed for use in laboratories.

\section{Acknowledgements}

The authors thank all ID26 staff and users for making excellent use of this instrument. The ESRF crystal analyzer laboratory is acknowledged (R. Verbeni, C. Lapras).

\section{References}

Ablett, J. M., Prieur, D., Céolin, D., Lassalle-Kaiser, B., Lebert, B., Sauvage, M., Moreno, T., Bac, S., Balédent, V., Ovono, A., Morand, 
M., Gélebart, F., Shukla, A. \& Rueff, J.-P. (2019). J. Synchrotron Rad. 26, 263-271.

Abraham, B., Nowak, S., Weninger, C., Armenta, R., Defever, J., Day, D., Carini, G., Nakahara, K., Gallo, A., Nelson, S., Nordlund, D., Kroll, T., Hunter, M. S., van Driel, T., Zhu, D., Weng, T.-C., AlonsoMori, R. \& Sokaras, D. (2019). J. Synchrotron Rad. 26, 629-634.

Alonso-Mori, R., Kern, J., Gildea, R. J., Sokaras, D., Weng, T. C., Lassalle-Kaiser, B., Tran, R., Hattne, J., Laksmono, H., Hellmich, J., Glöckner, C., Echols, N., Sierra, R. G., Schafer, D. W., Sellberg, J., Kenney, C., Herbst, R., Pines, J., Hart, P., Herrmann, S., GrosseKunstleve, R. W., Latimer, M. J., Fry, A. R., Messerschmidt, M. M., Miahnahri, A., Seibert, M. M., Zwart, P. H., White, W. E., Adams, P. D., Bogan, M. J., Boutet, S., Williams, G. J., Zouni, A., Messinger, J., Glatzel, P., Sauter, N. K., Yachandra, V. K., Yano, J. \& Bergmann, U. (2012). Proc. Natl Acad. Sci. USA, 109, 19103-19107.

Alonso-Mori, R., Sokaras, D., Zhu, D., Kroll, T., Chollet, M., Feng, Y., Glownia, J. M., Kern, J., Lemke, H. T., Nordlund, D., Robert, A., Sikorski, M., Song, S., Weng, T.-C. \& Bergmann, U. (2015). J. Synchrotron Rad. 22, 612-620.

Anwar, M. I., Ha, S. S., Hwang, B.-J., Han, S., Oh, M. S. H., Faiyaz, M., Noh, D. Y., Kang, H. C. \& Kim, S. (2019). J. Korean Phys. Soc. 75, 494-497.

Bergmann, U. \& Cramer, S. P. (1998). Proc. SPIE, 3448, 198.

Bokhoven, J. A. van \& Lamberti, C. (2016). X-ray Absorption and $X$-ray Emission Spectroscopy: Theory and Applications. Chichester: Wiley.

Bryzgunov, V. A. (2000). Tech. Phys. 45, 46-49.

Bunău, O. \& Joly, Y. (2009). J. Phys. Condens. Matter, 21, 345501.

Dickinson, B., Seidler, G. T., Webb, Z. W., Bradley, J. A., Nagle, K. P., Heald, S. M., Gordon, R. A. \& Chou, I. M. (2008). Rev. Sci. Instrum. 79, 123112 .

Dikaya, O., Nachtegaal, M., Szlachetko, J., Ebner, K., Saveleva, V., Weder, N., Probst, B., Alberto, R., Serebrennikov, D., Clementyev, E., Maksimova, K., Goikhman, A. \& Smolentsev, G. (2020). Results Phys. 18, 103212.

Doriese, W. B., Abbamonte, P., Alpert, B. K., Bennett, D. A., Denison, E. V., Fang, Y., Fischer, D. A., Fitzgerald, C. P., Fowler, J. W., Gard, J. D., Hays-Wehle, J. P., Hilton, G. C., Jaye, C., McChesney, J. L., Miaja-Avila, L., Morgan, K. M., Joe, Y. I., O’Neil, G. C., Reintsema, C. D., Rodolakis, F., Schmidt, D. R., Tatsuno, H., Uhlig, J., Vale, L. R., Ullom, J. N. \& Swetz, D. S. (2017). Rev. Sci. Instrum. 88, 053108.

Duan, P., Gu, S., Cao, H., Li, J. \& Huang, Y. (2017). X-ray Spectrom. 46, 12-18.

Ducotté, L., Glatzel, P., Marion, P., Lapras, C., Lesourd, M., Harris, A. \& Heyman, C. (2010). Diamond Light Source Proc. 1, e26.

Fister, T. T., Seidler, G. T., Wharton, L., Battle, A. R., Ellis, T. B., Cross, J. O., Macrander, A. T., Elam, W. T., Tyson, T. A. \& Qian, Q. (2006). Rev. Sci. Instrum. 77, 063901.

Glatzel, P., Bergmann, U., de Groot, F. M. F. \& Cramer, S. P. (2001). Phys. Rev. B, 64, 045109.

Glatzel, P., Weng, T. C. T.-C., Kvashnina, K., Swarbrick, J., Sikora, M., Gallo, E., Smolentsev, N. \& Mori, R. A. (2013). J. Electron Spectrosc. Relat. Phenom. 188, 17-25.

Groot, F. M. F. de \& Kotani, A. (2008). Core Level Spectroscopy of Solids. New York: Taylor and Francis.

Hiraoka, N., Fukui, H., Tanida, H., Toyokawa, H., Cai, Y. Q. \& Tsuei, K. D. (2013). J. Synchrotron Rad. 20, 266-271.

Holden, W. M., Hoidn, O. R., Ditter, A. S., Seidler, G. T., Kas, J., Stein, J. L., Cossairt, B. M., Kozimor, S. A., Guo, J., Ye, Y., Marcus, M. A. \& Fakra, S. (2017). Rev. Sci. Instrum. 88, 073904.

Honkanen, A.-P., Verbeni, R., Simonelli, L., Moretti Sala, M., Monaco, G. \& Huotari, S. (2014a). J. Synchrotron Rad. 21, 104-110.

Honkanen, A.-P., Verbeni, R., Simonelli, L., Moretti Sala, M., AlZein, A., Krisch, M., Monaco, G. \& Huotari, S. (2014b). J. Synchrotron Rad. 21, 762-767.

Huotari, S., Pylkkänen, T., Verbeni, R., Monaco, G. \& Hämäläinen, K. (2011). Nat. Mater. 10, 489-493.
Huotari, S., Sahle, C. J., Henriquet, C., Al-Zein, A., Martel, K., Simonelli, L., Verbeni, R., Gonzalez, H., Lagier, M.-C., Ponchut, C., Moretti Sala, M., Krisch, M. \& Monaco, G. (2017). J. Synchrotron Rad. 24, 521-530.

Huotari, S., Vankó, Gy., Albergamo, F., Ponchut, C., Graafsma, H., Henriquet, C., Verbeni, R. \& Monaco, G. (2005). J. Synchrotron Rad. 12, 467-472.

Jahrman, E. P., Holden, W. M., Ditter, A. S., Kozimor, S. A., Kihara, S. L. \& Seidler, G. T. (2019). Rev. Sci. Instrum. 90, 013106.

Kavčič, M. (2012). X-ray Spectroscopy, edited by S. K. Sharma, pp. 81-98. Rijeka: InTech.

Kavčič, M., Budnar, M., Mühleisen, A., Gasser, F., Žitnik, M., Bučar, K. \& Bohinc, R. (2012). Rev. Sci. Instrum. 83, 033113.

Kleymenov, E., Van Bokhoven, J. A. J. A., David, C., Glatzel, P., Janousch, M., Alonso-Mori, R., Studer, M., Willimann, M., Bergamaschi, A., Henrich, B. \& Nachtegaal, M. (2011). Rev. Sci. Instrum. 82, 065107.

Kotani, A. \& Shin, S. (2001). Rev. Mod. Phys. 73, 203-246.

Kowalska, J. K., Lima, F. A., Pollock, C. J., Rees, J. A. \& DeBeer, S. (2016). Isr. J. Chem. 56, 803-815.

Kuzmenko, D., Vogelsang, U., Hitz, S., Müller, D., Clark, A. H., Kinschel, D., Czapla-Masztafiak, J., Milne, C., Szlachetko, J. \& Nachtegaal, M. (2019). J. Anal. At. Spectrom. 34, 2105-2111.

Lafuerza, S., Carlantuono, A., Retegan, M. \& Glatzel, P. (2020). Inorg. Chem. 59, 12518-12535.

Lafuerza, S., Retegan, M., Detlefs, B., Chatterjee, R., Yachandra, V., Yano, J. \& Glatzel, P. (2020). Nanoscale, 12, 16270-16284.

Li, D., Alpert, B. K., Becker, D. T., Bennett, D. A., Carini, G. A., Cho, H. M., Doriese, W. B., Dusatko, J. E., Fowler, J. W., Frisch, J. C., Gard, J. D., Guillet, S., Hilton, G. C., Holmes, M. R., Irwin, K. D., Kotsubo, V., Lee, S. J., Mates, J. A. B., Morgan, K. M., Nakahara, K., Pappas, C. G., Reintsema, C. D., Schmidt, D. R., Smith, S. R., Swetz, D. S., Thayer, J. B., Titus, C. J., Ullom, J. N., Vale, L. R., Van Winkle, D. D., Wessels, A. \& Zhang, L. (2018). J. Low Temp. Phys. 193, 1287-1297.

Llorens, I., Lahera, E., Delnet, W., Proux, O., Braillard, A., Hazemann, J. L., Prat, A., Testemale, D., Dermigny, Q., Gelebart, F., Morand, M., Shukla, A., Bardou, N., Ulrich, O., Arnaud, S., Berar, J. F., Boudet, N., Caillot, B., Chaurand, P., Rose, J., Doelsch, E., Martin, P. \& Solari, P. L. (2012). Rev. Sci. Instrum. 83, 063104.

Machek, P., Welter, E., Caliebe, W., Brüggmann, U., Dräger, G. \& Fröba, M. (2007). AIP Conf. Proc. 879, 1755-1758.

Manceau, A., Enescu, M., Simionovici, A., Lanson, M., GonzalezRey, M., Rovezzi, M., Tucoulou, R., Glatzel, P., Nagy, K. L. \& Bourdineaud, J. P. (2016). Environ. Sci. Technol. 50, 1072110729.

Mao, W. L., Mao, H. K., Meng, Y., Eng, P. J., Hu, M. Y., Chow, P., Cai, Y. Q., Shu, J. \& Hemley, R. J. (2006). Science, 314, 636-638.

Miaja-Avila, L., O'Neil, G. C., Joe, Y. I., Alpert, B. K., Damrauer, N. H., Doriese, W. B., Fatur, S. M., Fowler, J. W., Hilton, G. C., Jimenez, R., Reintsema, C. D., Schmidt, D. R., Silverman, K. L., Swetz, D. S., Tatsuno, H. \& Ullom, J. N. (2016). Phys. Rev. X, 6, 031047 .

Moretti Sala, M., Martel, K., Henriquet, C., Al Zein, A., Simonelli, L., Sahle, C., Gonzalez, H., Lagier, M.-C., Ponchut, C., Huotari, S., Verbeni, R., Krisch, M. \& Monaco, G. (2018). J. Synchrotron Rad. 25, 580-591.

Mortensen, D. R. \& Seidler, G. T. (2017). J. Electron Spectrosc. Relat. Phenom. 215, 8-15.

Neese, F. (2018). WIREs Comput. Mol. Sci. 8, e1327.

Nowak, S. H., Armenta, R., Schwartz, C. P., Gallo, A., Abraham, B., Garcia-Esparza, A. T., Biasin, E., Prado, A., Maciel, A., Zhang, D., Day, D., Christensen, S., Kroll, T., Alonso-Mori, R., Nordlund, D., Weng, T. C. \& Sokaras, D. (2020). Rev. Sci. Instrum. 91, 033101.

Rehr, J. J., Kas, J. J., Vila, F. D., Prange, M. P. \& Jorissen, K. (2010). Phys. Chem. Chem. Phys. 12, 5503.

Retegan, M. (2020). Crispy v0.7.3, doi:10.5281/zenodo.1008184. 
Rovezzi, M., Harris, A., Detlefs, B., Bohdan, T., Svyazhin, A., Santambrogio, A., Degler, D., Baran, R., Reynier, B., Noguera Crespo, P., Heyman, C., Van Der Kleij, H.-P., Van Vaerenbergh, P., Marion, P., Vitoux, H., Lapras, C., Verbeni, R., Kocsis, M. M., Manceau, A. \& Glatzel, P. (2020). J. Synchrotron Rad. 27, 813-826.

Rovezzi, M., Lapras, C., Manceau, A., Glatzel, P. \& Verbeni, R. (2017). Rev. Sci. Instrum. 88, 013108.

Rueff, J.-P. P. \& Shukla, A. (2010). Rev. Mod. Phys. 82, 847-896.

Sa, J. (2014). High-Resolution XAS/XES. Boca Raton: CRC Press.

Schülke, W. (2007). Electron Dynamics by InelasticX-ray Scattering. Oxford University Press.

Smolentsev, G., Milne, C. J., Guda, A., Haldrup, K., Szlachetko, J., Azzaroli, N., Cirelli, C., Knopp, G., Bohinc, R., Menzi, S., Pamfilidis, G., Gashi, D., Beck, M., Mozzanica, A., James, D., Bacellar, C., Mancini, G. F., Tereshchenko, A., Shapovalov, V., Kwiatek, W. M., Czapla-Masztafiak, J., Cannizzo, A., Gazzetto, M., Sander, M., Levantino, M., Kabanova, V., Rychagova, E., Ketkov, S., Olaru, M., Beckmann, J. \& Vogt, M. (2020). Nat. Commun. 11, 2131.

Sokaras, D., Nordlund, D., Weng, T.-C. C., Mori, R. A., Velikov, P., Wenger, D., Garachtchenko, A., George, M., Borzenets, V., Johnson, B., Qian, Q., Rabedeau, T. \& Bergmann, U. (2012). Rev. Sci. Instrum. 83, 043112.

Sokaras, D., Weng, T.-C. C., Nordlund, D., Alonso-Mori, R., Velikov, P., Wenger, D., Garachtchenko, A., George, M., Borzenets, V., Johnson, B., Rabedeau, T. \& Bergmann, U. (2013). Rev. Sci. Instrum. 84, 053102.
Suortti, P., Buslaps, T., Fajardo, P., Honkimäki, V., Kretzschmer, M., Lienert, U., McCarthy, J. E., Renier, M., Shukla, A., Tschentscher, T. \& Meinander, T. (1999). J. Synchrotron Rad. 6, 69-80.

Szlachetko, J., Milne, C. J., Hoszowska, J., Dousse, J.-C., Błachucki, W., Sà, J., Kayser, Y., Messerschmidt, M., Abela, R., Boutet, S., David, C., Williams, G., Pajek, M., Patterson, B. D., Smolentsev, G., van Bokhoven, J. A. \& Nachtegaal, M. (2014). Struct. Dyn. 1, 021101 .

Szlachetko, J., Nachtegaal, M., Grolimund, D., Knopp, G., Peredkov, S., Czapla-Masztafiak, J. \& Milne, C. (2017). Appl. Sci. 7, 899.

Tu, M.-F., Doumy, G., Al Haddad, A., March, A. M., Southworth, S. H., Assoufid, L., Kumagai, Y., Walko, D. A., DiChiara, A. D., Liu, Z., Shi, B., Young, L. \& Bostedt, C. (2019). J. Synchrotron Rad. 26, 1956-1966.

Uruga, T., Tada, M., Sekizawa, O., Takagi, Y., Yokoyama, T. \& Iwasawa, Y. (2019). Chem. Rec. 19, 1444-1456.

Vankó, G., Bordage, A., Glatzel, P., Gallo, E., Rovezzi, M., Gawelda, W., Galler, A., Bressler, C., Doumy, G., March, A. M., Kanter, E. P., Young, L., Southworth, S. H., Canton, S. E., Uhlig, J., Smolentsev, G., Sundström, V., Haldrup, K., van Driel, T. B., Nielsen, M. M., Kjaer, K. S. \& Lemke, H. T. (2013). J. Electron Spectrosc. Relat. Phenom. 188, 166-171.

Wernet, P. (2019). Philos. Trans. R. Soc. A. 377, 201704.

Zimmermann, P., Peredkov, S., Abdala, P. M., DeBeer, S., Tromp, M., Müller, C. \& van Bokhoven, J. A. (2020). Coord. Chem. Rev. 423, 213466. 